\title{
Progressive increase in central nervous system immune activation in untreated primary HIV-1 infection
}

Joome Suh ${ }^{1 *}$, Elizabeth Sinclair ${ }^{2}$, Julia Peterson ${ }^{3}$, Evelyn Lee ${ }^{3}$, Tassos C Kyriakides ${ }^{4}$, Fang-yong Li ${ }^{4}$, Lars Hagberg ${ }^{5}$, Dietmar Fuchs ${ }^{6}$, Richard W Price ${ }^{3}$, Magnus Gisslen ${ }^{5}$ and Serena Spudich ${ }^{7}$

\begin{abstract}
Background: Central nervous system (CNS) inflammation is a mediator of brain injury in HIV infection. To study the natural course of CNS inflammation in the early phase of infection, we analyzed longitudinal levels of soluble and cellular markers of inflammation in cerebrospinal fluid (CSF) and blood, beginning with primary HIV-1 infection (PHI).

Methods: Antiretroviral-naïve subjects identified as having PHI (less than one year since HIV transmission) participated in phlebotomy and lumbar puncture at baseline and at variable intervals thereafter. Mixed-effects models were used to analyze longitudinal levels of CSF neopterin and percentages of activated cluster of differentiation (CD) $4+$ and CD8+ T-cells (co-expressing CD38 and human leukocyte antigen-D-related (HLA-DR)) in blood and CSF.

Results: A total of 81 subjects were enrolled at an average of 100 days after HIV transmission and had an average follow-up period of 321 days, with the number of visits ranging from one to 13. At baseline, the majority of subjects had CSF neopterin concentrations above the upper limit of normal. The baseline concentration was associated with the longitudinal trajectory of CSF neopterin. In subjects with baseline levels of less than $21 \mathrm{nmol} / \mathrm{L}$, a cutoff value obtained from a mixed-effects model, CSF neopterin increased by $2.9 \%$ per 10 weeks $(n=33 ; P<0.001)$, whereas it decreased by $6.7 \%$ in subjects with baseline levels of more than $21 \mathrm{nmol} / \mathrm{L}(\mathrm{n}=11 ; P=0.001)$. In a subset with available flow cytometry data $(n=42)$, the percentages of activated CD4+ and CD8+ T-cells in CSF increased by $0.8(P<0.001)$ and $0.73(P=0.02)$ per 10 weeks, respectively.
\end{abstract}

Conclusions: Neopterin levels and the percentages of activated CD4+ and CD8+ T-cells in CSF progressively increase in most subjects without treatment during early HIV-1 infection, suggesting an accrual of intrathecal inflammation, a major contributor to neuropathology in HIV infection.

Keywords: HIV, Central Nervous System Viral Infections, Inflammation, Neopterin, Activated T-cells, Immune Activation

\section{Background}

With the increasing availability of combination antiretroviral therapy (cART) the prevalence of HIV-associated dementia (HAD, the most severe form of HIV-associated neurocognitive disorder (HAND)) decreased from between 10 and $15 \%$ to about $2 \%$ in the HIV-1-infected population in the United States [1]. However, milder forms of HAND remain unchanged at a prevalence of between 40 and 50\% and are associated with decreased quality of life, poor treatment adherence, and higher mortality risk [2-4]. While

\footnotetext{
* Correspondence: joome.suh@yale.edu

'Yale School of Medicine, 367 Cedar Street, New Haven CT 06510, USA Full list of author information is available at the end of the article
}

poor viral suppression is a contributor to this high prevalence, neurocognitive impairment is also observed in individuals with adequate cART adherence and suppressed viral loads $[5,6]$ for reasons that are unclear. Studies have shown that inflammation, a mediator of central nervous system (CNS) injury [7], persists at a low level for many years despite cART [8-10], possibly causing continual damage to the CNS.

In exploring explanations for persistent CNS injury, some studies have focused on the impact of early infection prior to initiation of CART, since it is known that HIV enters the CNS and induces immune activation soon after transmission [11,12]. Emerging evidence from 
studies examining cerebrospinal fluid (CSF) and neuroimaging biomarkers in primary HIV infection (PHI, defined as within the first year of transmission) suggests that neuronal injury and dysfunction are already occurring in this stage [13-15]. These studies highlight the potential importance of the early stage of infection in HIV neuropathogenesis. Although cross-sectional studies have provided snapshots of processes occurring in the CNS during PHI [16], longitudinal processes occurring at this stage of infection in ART-naïve subjects have not been described.

In this study, we sought to examine the natural course of CNS inflammation starting in PHI. We hypothesized that CNS immune activation stimulated during initial infection would persist, and perhaps intensify, prior to initiation of ART, establishing a progressive inflammatory environment in the CNS. In a cohort of ART-naïve PHI subjects, we analyzed blood and CSF for longitudinal levels of soluble and cellular markers of inflammation. Neopterin was examined as a soluble surrogate biomarker of inflammation, and cluster of differentiation (CD)38 and human leukocyte antigen-D-related (HLA-DR) expressed together on CD4+ and CD8+ T-cells were examined as cellular markers of immune activation.

\section{Methods}

\section{Study participants}

ART-naïve HIV-1-infected subjects within one year of transmission were recruited in Gothenburg, Sweden $(\mathrm{n}=17)$ and San Francisco, United States $(n=64)$. As described previously [16], PHI in the participants was confirmed by a combination of seroconversion, nucleic acid testing, or lesssensitive enzyme-linked immunosorbent assay (ELISA) testing, according to the serologic testing algorithm for recent HIV seroconversion (STARHS) methods [17]. The duration since transmission was estimated by considering the date at onset of seroconversion symptoms as 14 days post-transmission [18]. For participants without seroconversion symptoms $(n=16)$, we estimated the date of transmission as halfway between the most recent negative and positive HIV tests [19]. Protocols were approved by the University of California San Francisco Committee on Human Research (H9133-26278) and the Research Ethics Committee of the University of Gothenburg (Ö588-01). Informed consent was obtained from all participants prior to enrollment.

\section{Data collection}

Longitudinal paired samples of CSF and blood were obtained prior to cART. In San Francisco, visits were scheduled at baseline (earliest enrollment after initial transmission), six weeks, and every subsequent six months from baseline, while in Gothenburg, study intervals were variable. Opportunistic infections and major neurological diseases such as stroke, multiple sclerosis, and seizure disorders were ruled out at baseline. Blood samples were analyzed for HIV RNA, albumin, CD4+ lymphocytes and CD8+ lymphocytes, and CSF samples were analyzed for HIV RNA, albumin, and white blood cell (WBC) count, as previously described [16]. Concentrations of CSF and blood neopterin were measured in previously frozen samples in the laboratory of Dr Fuchs by commercial immunoassays (BRAHMS Aktiengesellschaft, Hennigsdorf, Germany). A subset of fresh whole blood and CSF samples obtained in San Francisco $(n=42)$ was further analyzed by multiparameter flow cytometry as previously described [20,21] using FACSDiva (BD Biosciences, San Jose, USA) to measure the percentage of CD4+ and CD8+ cells with dual positivity for activation markers CD38 and HLA-DR. Data was analyzed with FlowJo (TreeStar, Ashland, Oregon, United States).

\section{Statistical analysis}

All statistical analyses were performed using SPSS version 19, IBM Corp., Armonk, USA. All descriptive statistics are presented as median and interquartile range (IQR) for continuous variables, and as frequency (\%) for categorical variables. Fischer's exact test, the Mann-Whitney U test, and Spearman's rank correlation coefficient were used where appropriate.

To analyze the longitudinal trajectory of CSF neopterin, we used mixed-effects models, which can accommodate differences among subjects in the total number of visits and intervals between visits. CSF neopterin concentrations were log-transformed to approximate a normal distribution before formal modeling. The models included fixed effects of baseline CSF neopterin and days post-transmission, and the random effect of intercept. The quadratic time effect was excluded from the final model because of statistical non-significance $(P=0.31)$. We also included an interaction term (days post-transmission $x$ baseline CSF neopterin) as a fixed effect to examine if the trajectory of CSF neopterin over days post-transmission depended on the baseline level. Since baseline CSF neopterin was used as a predictor, only CSF neopterin values from subsequent visits were used as observations of the dependent variable.

This model yielded a regression coefficient for slope (change in CSF neopterin per day post-transmission) equal to $0.000294-1.397 \mathrm{E}-5 \times$ baseline CSF neopterin, suggesting that the slope of CSF neopterin trajectory was dependent on baseline CSF neopterin. Based on this regression coefficient, baseline CSF neopterin values greater than $21 \mathrm{nmol} / \mathrm{L}$ yielded a negative slope, whereas baseline CSF neopterin values less than $21 \mathrm{nmol} / \mathrm{L}$ yielded a positive slope. Therefore, a baseline CSF neopterin level of $21 \mathrm{nmol} / \mathrm{L}$ was a cutoff value that determined the increasing versus decreasing trajectory of CSF neopterin. These results were confirmed by another model that used 
baseline CSF neopterin as a dichotomous variable (above or below $21 \mathrm{nmol} / \mathrm{L}$ ). Although only the group with baseline CSF neopterin below $21 \mathrm{nmol} / \mathrm{L}$ included subjects with visits beyond 150 weeks, sensitivity analysis constraining the time axis to 150 weeks yielded the same trajectories as above. Therefore the full analysis, which included these visits, is presented in this paper.

Similarly, we used mixed-effects models to examine trends in the proportions of CD4+ and CD8+ cells co-expressing CD38 and HLA-DR in blood and CSF. In each of the four models, days post-transmission was a fixed effect, intercept was a random effect, and the percentage of cells coexpressing CD38 and HLA-DR was the outcome variable.

\section{Results}

\section{Study participant characteristics}

The characteristics of participants are shown in Table 1. Subjects were mostly male, had a median age of 36 years (IQR: 28 to 45), and were at a median of 100 days posttransmission at baseline. Although the median blood CD4+ T-cell count was 552 cells/ $\mu \mathrm{L}$ (IQR: 389 to 720 ), one individual had an extremely low baseline CD4+ T-cell count of 111 cells $/ \mu \mathrm{L}$. The total number of visits ranged from 1 to 13, and the median duration of follow-up was 321 days.

\section{Baseline measures of immune activation}

Consistent with a previous report on a subset of this cohort [16], the median CSF neopterin concentration at baseline was $10.2 \mathrm{nmol} / \mathrm{L}$ (IQR: 6.6 to 21.3 ), with $81 \%$ of subjects

Table 1 Baseline demographic and laboratory features of subjects and follow-up duration

\begin{tabular}{|c|c|}
\hline Baseline feature/lab value & Median (interquartile range) \\
\hline Total number of subjects & 81 \\
\hline Male (\%) & $77(95.1)$ \\
\hline Age (years) & 36 (28 to 45$)$ \\
\hline $\begin{array}{l}\text { Duration since transmission at } \\
\text { baseline (days) }\end{array}$ & $100(54$ to 153$)$ \\
\hline Blood CD4+ T-cells (cells/uL) & 552 (389 to 720$)$ \\
\hline Plasma HIV RNA (log10 copies/mL) & 4.56 (3.97 to 5.18$)$ \\
\hline CSF HIV RNA (log10 copies/mL) & 2.77 (1.80 to 3.47$)$ \\
\hline Plasma neopterin (nmol/L) & 15.1 (9.4 to 21.6$)$ \\
\hline CSF neopterin (nmol/L) & $10.2(6.6$ to 21.3$)$ \\
\hline$\%$ Blood CD4 + CD38 + HLA-DR $+{ }^{a}$ & 7.5 (6.2 to 11.6$)$ \\
\hline$\%$ Blood CD8 + CD38 + HLA-DR $+{ }^{a}$ & $55.3(47.4$ to 68.1$)$ \\
\hline$\%$ CSF CD4 + CD38 + HLA-DR $+{ }^{a}$ & 14.2 (8.5 to 22.2$)$ \\
\hline$\%$ CSF CD8 + CD38 + HLA-DR + ${ }^{a}$ & 70.9 (60.9 to 80.6$)$ \\
\hline Total number of visits & 2 (1 to 3$)$ \\
\hline Duration of follow-up (days) & 321 (105 to 727 ) \\
\hline
\end{tabular}

${ }^{a} n=42$ for flow cytometry analysis. CD, cluster of differentiation; CSF, cerebrospinal fluid; HLA-DR, human leukocyte antigen-D-related. having levels above $5.8 \mathrm{nmol} / \mathrm{L}$, the upper limit of normal (ULN) [22]. Plasma neopterin at baseline was also elevated at $15.1 \mathrm{nmol} / \mathrm{L}$ (IQR: 9.4 to $21.6, \mathrm{ULN}: 8.7 \mathrm{nmol} / \mathrm{L}$ [23]). Percentages of activated CD4+ and CD8+ cells in both blood and CSF were also elevated compared to published values in HIV-uninfected subjects obtained using similar methods (Table 1) [20]. Plasma neopterin correlated positively with the percentage of activated CD4+ cells $\left(\mathrm{r}_{\mathrm{s}}=\right.$ 0.38, $P=0.02)$ and CD8+ cells $\left(r_{\mathrm{s}}=0.40, P=0.02\right)$ in blood, and the percentage of activated CD8+ cells in CSF $\left(\mathrm{r}_{\mathrm{s}}=\right.$ $0.41, P=0.01)$. CSF neopterin correlated moderately with the percentage of activated CD4+ cells $\left(r_{s}=0.36, P=0.03\right)$ and CD $8+$ cells $\left(r_{s}=0.37, P=0.03\right)$ in blood and strongly correlated with the percentage of activated CD8+ cells in $\operatorname{CSF}\left(\mathrm{r}_{\mathrm{s}}=0.63, P<0.001\right)$.

\section{Post-transmission trajectory of cerebrospinal fluid neopterin}

We next applied linear mixed-effects models to examine longitudinal changes in intrathecal immune activation prior to ART. A total of 44 subjects with a baseline CSF neopterin value and at least one follow-up value contributed to the analysis. Two significant trends were observed and depended on the baseline CSF neopterin concentration (interaction effect, $P<0.001$ ) being above or below $21 \mathrm{nmol} / \mathrm{L}$, a cutoff value obtained from a mixed-effects model (see Methods). In participants with baseline CSF neopterin concentrations below $21 \mathrm{nmol} / \mathrm{L}$, henceforth referred to as the 'low group' ( $n=33)$, CSF neopterin levels increased over time, and a lower baseline value was associated with a steeper increase. This group included eight subjects with baseline CSF neopterin concentrations within the normal range. In contrast, CSF neopterin decreased in the minority of participants with baseline CSF neopterin concentrations above $21 \mathrm{nmol} / \mathrm{L}$, henceforth referred to as the 'high group' $(n=11)$. Within this group, a higher baseline value was associated with a faster decline.

To confirm the observation of $21 \mathrm{nmol} / \mathrm{L}$ as a cutoff value, we ran a similar mixed-effects model with baseline CSF neopterin as a categorical variable. This model yielded the average change in CSF neopterin level over time separately for the low and high groups, and showed that they were significantly different as evidenced by the group-by-time interaction $(P<0.001)$. As shown in Figure 1, CSF neopterin concentration increased in the low group by approximately $2.9 \%$ per 10 weeks $(P<0.001)$, and decreased in the smaller high group by approximately $6.7 \%$ per 10 weeks $(P=0.001)$.

\section{Correlations with high and low baseline cerebrospinal fluid neopterin levels}

When the groups with baseline CSF neopterin concentrations below and above $21 \mathrm{nmol} / \mathrm{L}$ were compared on 


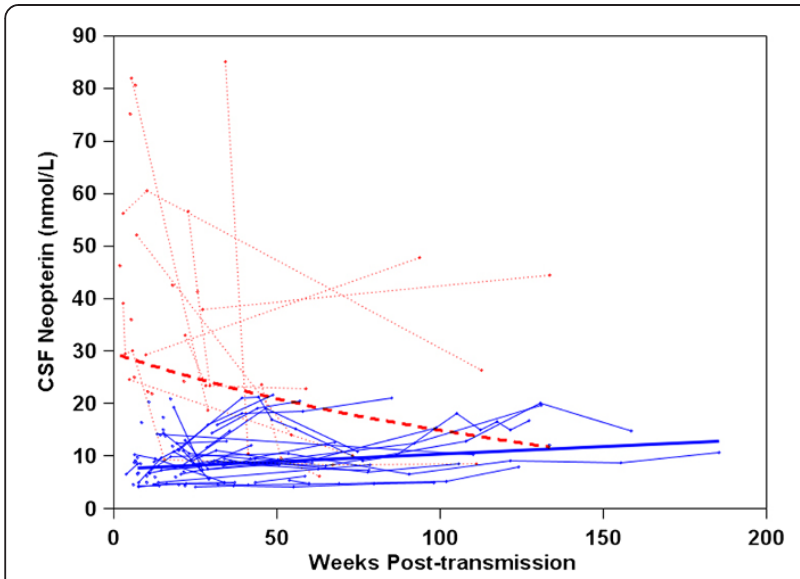

Figure 1 Trajectories of cerebrospinal fluid (CSF) neopterin in early HIV-1 infection. Thin lines represent the trajectories of individual subjects and bold lines represent the average slopes of high (red) and low (blue) groups (see text).

baseline parameters (Table 2), significant differences were not found in age or days post-transmission. However, the high group was found to have a lower CD4+ cell count in blood $(P<0.001)$, higher neopterin levels in plasma $(P=0.006)$ and CSF $(P<0.001)$, and HIV RNA levels in both plasma $(P=0.02)$ and CSF $(P=0.006)$ that were greater by more than an order of magnitude. In both groups, the level of HIV RNA in CSF was lower than that found in plasma.

Despite a higher CSF-to-plasma neopterin ratio in the high group (Figure 2A, $P<0.001$ ), the two groups did not differ in the CSF-to-plasma HIV RNA ratio (Figure 2B, $P=0.37$ ) or the CSF-to-plasma albumin ratio (Figure $2 \mathrm{C}$, $P=0.06)$. The neopterin-to-HIV RNA ratio was higher in the high group in CSF $(P<0.001)$ but not in plasma $(P=0.10)$.
Clinically, two out of 33 subjects in the low group (5.9\%) and one out of 11 subjects in the high group (9.1\%) had specific neurological seroconversion symptoms (excluding headache). The two neurosymptomatic patients in the low group had Guillain-Barré-like syndrome and meningitis, and the one patient in the high group had meningitis.

\section{Post-transmission trajectory of activated CD4+ and CD8+ T-cells in blood and cerebrospinal fluid}

Mixed-model analyses of subjects with available flow cytometry data $(n=42)$ showed an increasing trend in the percentage of activated CD4+ cells in blood at a rate of 0.23 per 10 weeks $(P=0.05$, Figure $3 \mathrm{~A})$. A significant trend for activated CD8+ cells in blood was not found $(P=0.54$, Figure $3 \mathrm{~B})$. In CSF, the percentage of activated $\mathrm{CD} 4+$ cells increased at a rate of 0.63 per 10 weeks $(P=0.04)$. We noted an outlier value that had a sharp increase from 8 to $54 \%$ from day 26 to 51 post-transmission; re-analysis excluding this outlier yielded a rate of 0.8 per 10 weeks $(P<0.001$, Figure $3 C)$. The percentage of activated CD8+ cells in CSF increased at a rate of 0.73 per 10 weeks $(P=0.02$, Figure 3D).

\section{Discussion}

In this study, we analyzed the longitudinal levels of CSF neopterin and percentages of activated CD4+ and CD8+ T-cells in blood and CSF, reporting for the first time the natural history of change in these measures of immune activation prior to cART beginning in PHI. HLA-DR and CD38 were used as markers of T-cell activation, and CSF neopterin was used as a soluble marker of CNS inflammation, as it is produced by activated macrophages and astrocytes mainly in response to interferon-gamma. It has been shown that $97.5 \%$ of neopterin in CSF is

Table 2 Baseline parameter comparisons of low and high groups

\begin{tabular}{|c|c|c|c|}
\hline & $\begin{array}{l}\text { Low group (baseline CSF } \\
\text { neopterin }<21, n=33 \text { ) }\end{array}$ & $\begin{array}{l}\text { High group (baseline CSF } \\
\text { neopterin }>21, n=11 \text { ) }\end{array}$ & $P$ value \\
\hline Age (years) & 37 (30 to 46$)$ & 36 (28 to 47$)$ & 0.87 \\
\hline Days post-transmission & 136 (84 to 189$)$ & 70 (34 to 191$)$ & 0.23 \\
\hline Blood CD4+ T-cells (cells/ $\mu \mathrm{L}$ ) & 601 (444 to 750$)$ & 308 (275 to 389 ) & $<0.001$ \\
\hline Blood CD8+ T-cells (cells/ $\mu \mathrm{L})$ & $993(692$ to 1,269$)$ & $1,037(725$ to 1,707$)$ & 0.58 \\
\hline Plasma HIV RNA (log10 copies/mL) & 4.2 (3.8 to 4.8$)$ & $5.3(4.4$ to 5.9$)$ & 0.02 \\
\hline CSF HIV RNA (log10 copies/mL) & 2.5 (1.7 to 3.2$)$ & 3.7 (2.8 to 4.9$)$ & 0.006 \\
\hline CSF:plasma HIV RNA ratio $(\log 10)$ & $-1.8(-2.2$ to -1.2$)$ & $-1.4(-2.3$ to -0.5$)$ & 0.37 \\
\hline CSF WBC count & $6(2.5$ to 9.5$)$ & $10(1$ to 11$)$ & 0.61 \\
\hline Plasma neopterin (nmol/L) & 13.9 (10.2 to 18.2$)$ & $21.5(18.3$ to 37.5$)$ & 0.006 \\
\hline CSF neopterin (nmol/L) & 8.5 (5.8 to 11.8$)$ & 39.1 (29.2 to 56.6$)$ & $<0.001$ \\
\hline CSF:plasma neopterin ratio & 0.66 (0.42 to 0.8$)$ & 1.8 (1.3 to 2.6$)$ & $<0.001$ \\
\hline CSF:plasma albumin ratio & 5.4 (4.3 to 6.4$)$ & $7.4(4.6$ to 10.9$)$ & 0.06 \\
\hline
\end{tabular}




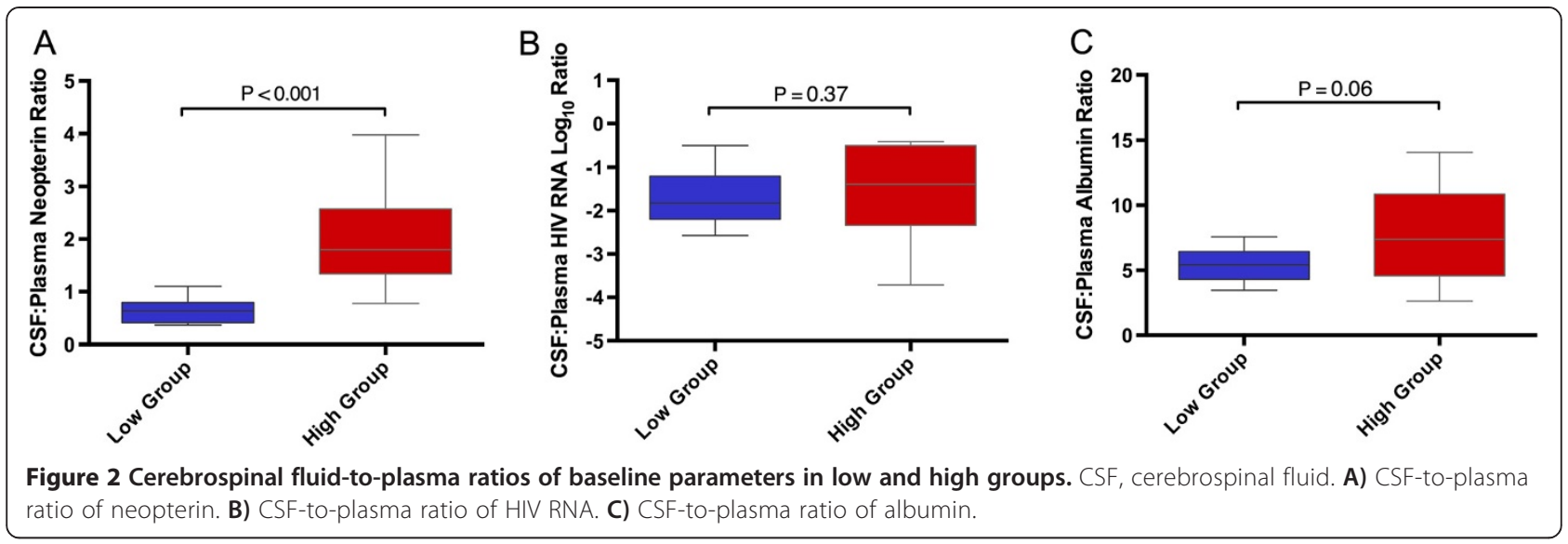

intrathecally produced [24], and it is therefore not simply a spillover from blood, but reflects intrathecal inflammation. Although with any CSF marker there is a concern that it may not accurately reflect processes in the brain, CSF neopterin has been frequently used for the reasons mentioned above as a surrogate biomarker of CNS inflammation and studied as a potential ancillary diagnostic marker for HAD $[22,25]$. Additionally, CSF neopterin has previously been shown in this PHI cohort to correlate with neurofilament light chain [13], a marker of neuronal injury.

Our analysis showed two trajectories of CSF neopterin beginning in PHI that were dependent on the baseline concentration of CSF neopterin. In the majority of participants, a slow increase was observed, similar to trends in chronic infection [26,27]. Previously unobserved was a downward trend in the quarter of the subjects who had baseline CSF neopterin concentrations above $21 \mathrm{nmol} / \mathrm{L}$,
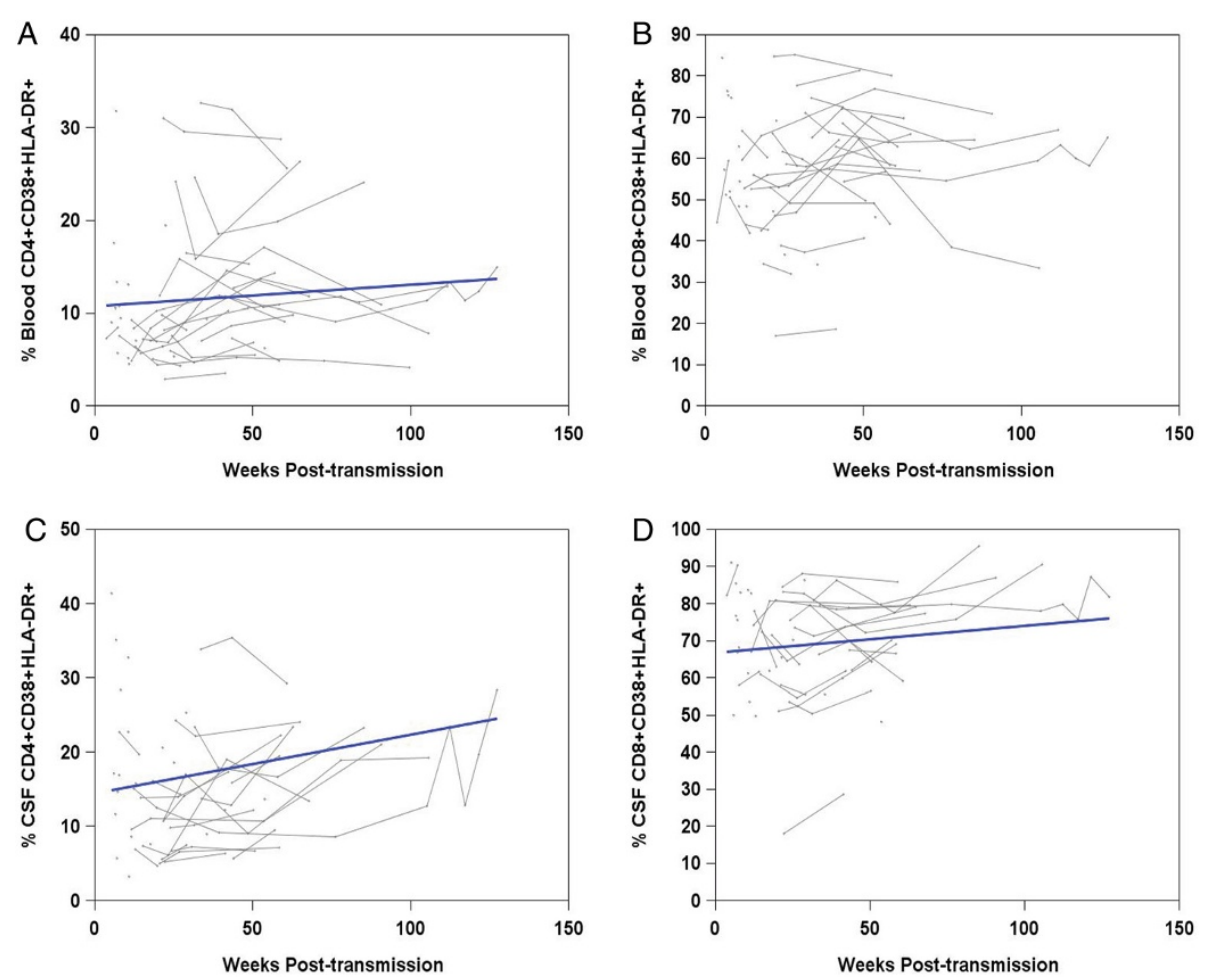

Figure 3 Trajectories of percentages of activated T-cells in blood and CSF in early HIV-1 infection. Thin lines represent the trajectories of individual subjects and bold lines represent average slopes obtained from mixed-effects models. A) Percentage of CD4+ CD38+ HLA-DR+ cells in blood. B) Percentage of CD8+ CD38+ HLA-DR+ cells in blood. No significant trend was observed. C) Percentage of CD4+ CD38+ HLA-DR+ cells in CSF. D) Percentage of CD8+ CD38+ HLA-DR+ cells in CSF. CD, cluster of differentiation; CSF, cerebrospinal fluid; HLA-DR, human leukocyte antigen-D-related. 
a value obtained through modeling. The median baseline CSF neopterin level in these subjects was more than sixfold greater than the upper limit of normal. Although CSF neopterin decreased over time, the rate was not nearly fast enough for a reduction in CSF neopterin to normal levels within the observed time interval.

To better characterize the groups, we compared them on multiple baseline parameters. In both groups, CSFto-plasma HIV RNA ratio was lower than the 1 log reduction typically found during chronic infection, as has been previously reported in early HIV infection $[11,16]$. However, viral load and neopterin concentration in both plasma and CSF were higher in the high group, suggesting a greater degree of systemic and intrathecal inflammation in the initial stage of infection.

Interestingly, despite a lower viral load in CSF compared to plasma in both groups, the concentration of neopterin was higher in CSF than in plasma in the high group, while the converse was true for the low group. Accordingly, a higher CSF-to-plasma neopterin ratio was observed in the high group even though the groups had similar CSF-to-plasma HIV RNA ratios. Further investigation revealed a higher neopterin-to-HIV RNA ratio only in the CSF of the high group, suggesting an amplified intrathecal immune response to the CSF viral load in this group.

Additionally in this study, we analyzed changes in the percentages of activated CD4+ and CD8+ T-cells in blood and CSF, beginning in PHI. A significant trend was not observed for blood CD8+ CD38+ HLA-DR+ cells, a subset that has been associated with the rate of $\mathrm{CD} 4+$ cell decline and prognosis in chronic infection [28-30]. However, in CSF, there was an increase in the proportion of activated CD8+ cells. Although associations with HAND in humans are yet unknown, activated CD8+ cells have been shown to produce cytolytic and pro-inflammatory molecules in the brains of macaques with CNS dysfunction from simian immunodeficiency virus (SIV) infection [31]. This observation corroborates the CSF neopterin findings of increasing CNS immune activation beginning in PHI prior to the typical initiation of antiretroviral treatment. The similar upward trajectories for neopterin and activated CD8+ cells in CSF are unsurprising as they were strongly correlated at baseline, consistent with previous reports [20]. These results highlight the utility of CSF neopterin as a marker of CNS inflammation for future studies.

Regarding the percentage of activated CD4+ T-cells in blood, an upward trend was observed in agreement with a previous report of an increase in the density of CD38 on CD4+ cells in blood during early infection [28]. A novel finding in this study was that the percentage of activated CD4+ T-cells also progressively increases in CSF beginning in early infection, which may have implications for the establishment of HIV replication within the CNS compartment, as activated CD4+ cells support viral replication more efficiently compared to resting T-cells $[32,33]$.

\section{Conclusions}

Our longitudinal analyses of both soluble and cellular measures of immune activation indicate that without treatment, CNS inflammation increases during primary HIV-1 infection in most individuals. In a minority of subjects with significantly higher levels of CSF neopterin at baseline, neopterin levels decrease, but only at a slow rate such that elevated levels persist throughout the follow-up period. These results are disconcerting because CNS inflammation and immune activation are considered to be major contributors to neurodegeneration in HIV infection $[7,34]$.

cART dramatically reduces the level of immune activation in blood and CSF [20,35-37], although elevated levels of neopterin and activated T-cells can persist in CSF [38]. Yilmaz et al. reported that the set-point of CSF neopterin in subjects on virologically suppressive cART is associated with the pre-ART level of CNS inflammation [39]. Therefore, our observation of a steady increase in CNS inflammation in the majority of subjects highlights the importance of identifying individuals early after infection, and supports prompt initiation of therapy to reduce CNS inflammation in PHI and achieve the lowest possible set-point on cART.

\section{Abbreviations}

CART: combined antiretroviral therapy; CD: cluster of differentiation; CNS: central nervous system; CSF: cerebrospinal fluid; ELISA: enzyme-linked immunosorbent assay; HAD: HIV-associated dementia; HIV: human immunodeficiency virus; HLA-DR: human leukocyte antigen-D-related; HAND: HIV-associated neurocognitive disorder; IQR: interquartile range; PHI: primary HIV infection; SIV: simian immunodeficiency virus; STARHS: serologic testing algorithm for recent HIV seroconversion; ULN: upper limit of normal; WBC: white blood cell.

\section{Competing interests}

The authors report no conflicts of interest associated with this work, with the following exceptions. TCK reports receiving statistical consulting fees from Yasoo Health Inc. (Jonesborough, USA). RWP reports receiving honorarium as speaker from Abbott (Abbott Park, USA). LH reports receiving personal compensation from Baxter (Deerfield, USA) and Meda (Solna, Sweden) for being on the advisory board, from Astella (Northbrook, USA) for speaking, and from the Scandinavian Journal Infectious Diseases as associated editor. MG reports receiving honoraria as speaker and/or advisor from AbbVie (North Chicago, USA), Bristol-Myers Squibb (Wallingford Center, USA), Gilead Sciences (Branford, USA), GlaxoSmithKline (Brentford, USA), Janssen-Cilag (Beerse, Belgium) and Merck (Whitehouse Station, USA). SS reports receiving travel support and an honorarium as speaker from AbbVie (North Chicago, USA) for a presentation at a scientific meeting.

\section{Authors' contributions}

JS: study conception, data analysis, and wrote the manuscript. ES: flow cytometry methods and analysis. JP: data collection. EL: data collection. TK: study design and edited the manuscript. FL: data analysis, created the figures, and edited the manuscript. LH: edited the manuscript. DF: neopterin methods and edited the manuscript. RP: study conception and data collection. MG: study conception, data collection, and edited the manuscript. 
SS: study conception, data collection, and edited the manuscript. All authors read and approved the manuscript.

\section{Acknowledgements}

The authors thank the generous participants who volunteered for these studies and members of the Core Immunology Laboratory (CIL) at the San Francisco General Hospital, the University of California San Francisco (UCSF) Options Study, and the Magnet/San Francisco AIDS Foundation for collaboration and referral of study subjects.

This work was supported by the National Institutes of Health (grants R01MH081772, K23MH074466, R01 NS043103, P01A1071713, M01RR00083), the UCSF AIDS Research Institute, the UCSF Academic Senate, the UCSF Research Evaluation and Allocation Committee, the Sahlgrenska Academy at University of Gothenburg (project number: ALFGBG-11067), the Swedish Research Council (project number: 2007-7092), and the GD Hsiung, PhD Medical Student Research Fellowship.

\section{Author details}

${ }^{1}$ Yale School of Medicine, 367 Cedar Street, New Haven CT 06510, USA. 2Department of Medicine, UCSF School of Medicine, 1001 Potrero Avenue, SFGH 100, San Francisco, CA 94143, USA. ${ }^{3}$ Department of Neurology, UCSF School of Medicine, 1001 Potrero Avenue, SFGH 1, San Francisco, CA 94110, USA. ${ }^{4}$ Yale Center for Analytical Sciences, Yale University, 300 George Street, Suite 555, New Haven CT 06520, USA. ${ }^{5}$ Department of Infectious Diseases, University of Gothenburg, Journalvägen 10, 41685 Gothenburg, Sweden. ${ }^{6}$ Division of Biological Chemistry, Innsbruck Medical University, Innrain 80, 6020 Innsbruck, Austria. ${ }^{7}$ Department of Neurology, Yale School of Medicine, 300 George Street, Room 8300c, New Haven CT 06510, USA.

Received: 16 July 2014 Accepted: 11 November 2014

Published online: 03 December 2014

\section{References}

1. Heaton RK, Clifford DB, Franklin DR, Woods SP, Ake C, Vaida F, Ellis RJ, Letendre SL, Marcotte TD, Atkinson JH, Rivera-Mindt M, Vigil OR, Taylor MJ, Collier AC, Marra CM, Gelman BB, McArthur JC, Morgello S, Simpson DM, McCutchan JA, Abramson I, Gamst A, Fennema-Notestine C, Jernigan TL, Wong J, Grant I: HIV-associated neurocognitive disorders persist in the era of potent antiretroviral therapy CHARTER Study. Neurology 2010, 75:2087-2096.

2. Hinkin CH, Castellon SA, Durvasula RS, Hardy DJ, Lam MN, Mason KI, Thrasher D, Goetz MB, Stefaniak M: Medication adherence among HIV + adults: effects of cognitive dysfunction and regimen complexity. Neurology 2002, 59:1944-1950.

3. Heaton RK, Marcotte TD, Mindt MR, Sadek J, Moore DJ, Bentley H, McCutchan JA, Reicks C, Grant I: The impact of HIV-associated neuropsychological impairment on everyday functioning. J Int Neuropsychol Soc 2004, 10:317-331.

4. Ellis RJ, Deutsch R, Heaton RK, Marcotte TD, McCutchan JA, Nelson JA, Abramson I, Thal LJ, Atkinson JH, Wallace MR, Grant I: Neurocognitive impairment is an independent risk factor for death in HIV infection. San Diego HIV Neurobehavioral Research Center Group. Arch Neurol 1997, 54:416-424.

5. Tozzi V, Balestra P, Bellagamba R, Corpolongo A, Salvatori MF, Visco-Comandini U, Vlassi C, Giulianelli M, Galgani S, Antinori A, Narciso P: Persistence of neuropsychologic deficits despite long-term highly active antiretrovira therapy in patients with HIV-related neurocognitive impairment: prevalence and risk factors. J Acquir Immune Defic Syndr 2007, 45:174-182.

6. Cysique LA, Maruff P, Brew BJ: Variable benefit in neuropsychological function in HIV-infected HAART-treated patients. Neurology 2006, 66:1447-1450.

7. Gonzalez-Scarano F, Martin-Garcia J: The neuropathogenesis of AIDS Nat Rev Immunol 2005, 5:69-81.

8. Abdulle S, Hagberg L, Svennerholm B, Fuchs D, Gisslen M: Continuing intrathecal immunoactivation despite two years of effective antiretroviral therapy against HIV-1 infection. AIDS 2002, 16:2145-2149.

9. Yilmaz A, Price RW, Spudich S, Fuchs D, Hagberg L, Gisslen M: Persistent intrathecal immune activation in HIV-1-infected individuals on antiretroviral therapy. J Acquir Immune Defic Syndr 2008, 47:168-173.

10. Eden A, Price RW, Spudich S, Fuchs D, Hagberg L, Gisslen M: Immune activation of the central nervous system is still present after $>4$ years of effective highly active antiretroviral therapy. J Infect Dis 2007, 196:1779-1783.
11. Valcour V, Chalermchai T, Sailasuta N, Marovich M, Lerdlum S, Suttichom D, Suwanwela NC, Jagodzinski L, Michael N, Spudich S, van Griensven F, de Souza M, Kim J, Ananworanich J: Central nervous system viral invasion and inflammation during acute HIV infection. J Infect Dis 2012, 206:275-282

12. Resnick L, Berger JR, Shapshak P, Tourtellotte WW: Early penetration of the blood-brain-barrier by HIV. Neurology 1988, 38:9-14

13. Peluso MJ, Meyerhoff DJ, Price RW, Peterson J, Lee E, Young AC, Walter R, Fuchs D, Brew BJ, Cinque P, Robertson K, Hagberg L, Zetterberg H, Gisslen M, Spudich S: Cerebrospinal fluid and neuroimaging biomarker abnormalities suggest early neurological injury in a subset of individuals during primary HIV infection. J Infect Dis 2013, 207:1703-1712.

14. Ragin A, Wu Y, Du H, Ochs R, Epstein L: Injury to the Brain is Evident Early in HIV Infection. In 18th Conference on Retroviruses and Opportunistic Infections (CROI). February 27-March 2, 2011. Boston. Abstract 55LB.

15. Lentz MR, Kim WK, Lee V, Bazner S, Halpern EF, Venna N, Williams K, Rosenberg ES, Gonzalez RG: Changes in MRS neuronal markers and T cell phenotypes observed during early HIV infection. Neurology 2009, 72:1465-1472.

16. Spudich S, Gisslen M, Hagberg L, Lee E, Liegler T, Brew B, Fuchs D, Tambussi G, Cinque $P$, Hecht FM, Price RW: Central nervous system immune activation characterizes primary human immunodeficiency virus 1 infection even in participants with minimal cerebrospinal fluid viral burden. J Infect Dis 2011, 204:753-760.

17. Zetola NM, Pilcher CD: Diagnosis and management of acute HIV infection. Infect Dis Clin North Am 2007, 21:19-48.

18. Lindback S, Thorstensson R, Karlsson AC, von Sydow M, Flamholc L, Blaxhult A, Sonnerborg A, Biberfeld G, Gaines H: Diagnosis of primary HIV-1 infection and duration of follow-up after HIV exposure. Karolinska Institute Primary HIV Infection Study Group. AIDS 2000, 14:2333-2339.

19. Little SJ, Frost SD, Wong JK, Smith DM, Pond SL, Ignacio CC, Parkin NT, Petropoulos CJ, Richman DD: Persistence of transmitted drug resistance among subjects with primary human immunodeficiency virus infection. J Virol 2008, 82:5510-5518.

20. Sinclair E, Ronquillo R, Lollo N, Deeks SG, Hunt P, Yiannoutsos CT, Spudich S, Price RW: Antiretroviral treatment effect on immune activation reduces cerebrospinal fluid HIV-1 infection. J Acquir Immune Defic Syndr 2008, 47:544-552.

21. Dahl V, Lee E, Peterson J, Spudich SS, Leppla I, Sinclair E, Fuchs D, Palmer S, Price RW: Raltegravir treatment intensification does not alter cerebrospinal fluid HIV-1 infection or immunoactivation in subjects on suppressive therapy. J Infect Dis 2011, 204:1936-1945.

22. Hagberg $L$, Cinque $P$, Gisslen M, Brew BJ, Spudich S, Bestetti A, Price RW, Fuchs D: Cerebrospinal fluid neopterin: an informative biomarker of central nervous system immune activation in HIV-1 infection. AIDS Res Ther 2010, 7:15.

23. Werner ER, Bichler A, Daxenbichler G, Fuchs D, Fuith LC, Hausen A, Hetzel H, Reibnegger $\mathrm{G}$, Wachter $\mathrm{H}$ : Determination of neopterin in serum and urine. Clin Chem 1987, 33:62-66.

24. Bogner JR, Jungehulsing B, Kronawitter U, Sadri I, Matuschke A, Goebel FD: Expansion of neopterin and beta-2-microglobulin in cerebrospinal-fluid reaches maximum levels early and late in the course of humanimmunodeficiency-virus Infection. Clin Investig 1992, 70:665-669.

25. Price RW, Epstein LG, Becker JT, Cinque P, Gisslen M, Pulliam L, MCArthur JC: Biomarkers of HIV-1 CNS infection and injury. Neurology 2007, 69:1781-1788

26. Gisslen M, Chiodi F, Fuchs D, Norkrans G, Svennerholm B, Wachter H, Hagberg L: Markers of immune stimulation in the cerebrospinal fluid during HIV infection: a longitudinal study. Scand J Infect Dis 1994, 26:523-533.

27. Gisslen M, Hagberg L, Fuchs D, Norkrans G, Svennerholm B: Cerebrospinal fluid viral load in HIV-1-infected patients without antiretroviral treatment - A longitudinal study. J Acquir Immune Defic Syndr 1998, 17:291-295

28. Deeks SG, Kitchen CM, Liu L, Guo H, Gascon R, Narvaez AB, Hunt P, Martin JN, Kahn JO, Levy J, McGrath MS, Hecht FM: Immune activation set point during early HIV infection predicts subsequent CD4+ T-cell changes independent of viral load. Blood 2004, 104:942-947.

29. Giorgi JV, Hultin LE, McKeating JA, Johnson TD, Owens B, Jacobson LP, Shih R, Lewis J, Wiley DJ, Phair JP, Wolinsky SM, Detels R: Shorter survival in advanced human immunodeficiency virus type 1 infection is more closely associated with T lymphocyte activation than with 
plasma virus burden or virus chemokine coreceptor usage. $J$ Infect Dis 1999, 179:859-870.

30. Giorgi JV, Liu Z, Hultin LE, Cumberland WG, Hennessey K, Detels R: Elevated levels of CD38+CD8+ T cells in HIV infection add to the prognostic value of low CD4+ T cell levels: results of 6 years of follow-up. The Los Angeles Center, Multicenter AIDS Cohort Study. J Acquir Immune Defic Syndr 1993, 6:904-912.

31. Marcondes MC, Burudi EM, Huitron-Resendiz S, Sanchez-Alavez M, Watry D, Zandonatti M, Henriksen SJ, Fox HS: Highly activated CD8 (+) T cells in the brain correlate with early central nervous system dysfunction in simian immunodeficiency virus infection. J Immunol 2001, 167:5429-5438.

32. Pan X, Baldauf HM, Keppler OT, Fackler OT: Restrictions to HIV-1 replication in resting CD4+ T lymphocytes. Cell Res 2013, 23:876-885.

33. Dolei A, Biolchini A, Serra C, Curreli S, Gomes E, Dianzani F: Increased replication of T-cell-tropic HIV strains and CXC-chemokine receptor-4 induction in T cells treated with macrophage inflammatory protein (MIP)-1alpha, MIP-1beta and RANTES beta-chemokines. AIDS 1998, 12:183-190

34. Spudich S, Gonzalez-Scarano F: HIV-1-related central nervous system disease: current issues in pathogenesis, diagnosis, and treatment. Cold Spring Harb Perspect Med 2012, 2:a007120.

35. Benito JM, Lopez M, Lozano S, Martinez P, Gonzalez-Lahoz J, Soriano V: CD38 expression on CD8 T lymphocytes as a marker of residual virus replication in chronically HIV-infected patients receiving antiretroviral therapy. AIDS Res Hum Retroviruses 2004, 20:227-233.

36. Gisslen M, Hagberg L: Antiretroviral treatment of central nervous system HIV-1 infection: a review. HIV Med 2001, 2:97-104.

37. Spudich S, Lollo N, Liegler T, Deeks SG, Price RW: Treatment benefit on cerebrospinal fluid HIV-1 levels in the setting of systemic virological suppression and failure. J Infect Dis 2006, 194:1686-1696.

38. Neuenburg JK, Cho TA, Nilsson A, Bredt BM, Hebert SJ, Grant RM, Price RW: T-cell activation and memory phenotypes in cerebrospinal fluid during HIV infection. J Acquir Immune Defic Syndr 2005, 39:16-22.

39. Yilmaz A, Yiannoutsos CT, Fuchs D, Price RW, Crozier K, Hagberg L, Spudich S, Gisslen M: Cerebrospinal fluid neopterin decay characteristics after initiation of antiretroviral therapy. J Neuroinflammation 2013, 10:62.

Cite this article as: Suh et al:: Progressive increase in central nervous system immune activation in untreated primary HIV-1 infection.

Journal of Neuroinflammation 2014 11:199.

\section{Submit your next manuscript to BioMed Central and take full advantage of:}

- Convenient online submission

- Thorough peer review

- No space constraints or color figure charges

- Immediate publication on acceptance

- Inclusion in PubMed, CAS, Scopus and Google Scholar

- Research which is freely available for redistribution 